\title{
Effects of Friction and Anvil Design on Plastic Deformation during the Compression Stage of High-Pressure Torsion
}

\author{
Yuepeng Song ${ }^{1,2,3, *}$, Miaomiao Chen ${ }^{1,2}$, Baoyan $\mathrm{Xu}^{1,2}$, Dongsheng $\mathrm{Gao}^{2}$, Jing $\mathrm{Guo}^{1,2,3}$, Lingfeng $\mathrm{Xu}^{1,2}$, \\ Zheng Wang ${ }^{1,2}$, and Hyoung Seop Kim ${ }^{3, *}$ \\ ${ }^{I}$ Shandong Agricultural University, Mechanical and Electronic Engineering College, Tai'an 271018, China \\ ${ }^{2}$ Shandong Agricultural University, Shandong Provincial Key Laboratory of Horticultural Machineries and Equipments, \\ Tai'an, China \\ ${ }^{3}$ Pohang University of Science and Technology, Department of Materials Science and Engineering, Pohang 37673, \\ Republic of Korea
}

\begin{abstract}
Herein, we report the results of our investigation on the effect of friction and anvil design on the heterogeneous plastic-deformation characteristics of copper during the compressive stage of high-pressure torsion (HPT), using the finite element method. The results indicate that the friction and anvil geometry play important roles in the homogeneity of the deformation. These variables affect the heterogeneous level of strain in the HPT compressed disks, as well as the flash in the disk edge region. The heterogeneous plastic deformation of the disks becomes more severe with the increasing depth of the cavity, as anvil angle and friction coefficient increase. However, the homogeneity increases with increases in the wall angle. The length of flash and the area of the dead metal zone increase with the depth of the cavity, while they decrease at a wall angle of $180^{\circ}$.
\end{abstract}

(Received March 17, 2016; Accepted May 25, 2016)

Keywords: high pressure torsion, copper, deformation, finite element analysis, anvil structure

\section{INTRODUCTION}

Severe plastic deformation (SPD) processes have been studied extensively and used as convenient methods to manufacture ultrafine-grained, nanostructured metals and their alloys. SPD processes currently being used include accumulative roll bonding (ARB), equal-channel angular pressing (ECAP), and high-pressure torsion (HPT) [1-4]. Among these SPD methods, the HPT process is particularly noteworthy because it can produce finer grains, with a higher fraction of high-angle grain boundaries, than can the other SPD methods [4-9].

The concept of the HPT process was first proposed over 70 years ago by Bridgman, but the process has been intensively investigated with respect to grain refinement within the last 10 years [4-6]. The HPT process consists of two stages: the compression and compression-torsion stages. In the initial compression stage, high pressure (up to $10 \mathrm{GPa}$ ) is applied to a sample placed in between the top and bottom dies. After

* Corresponding Author: Hyoung Seop Kim, Yuepeng Song [Tel: +82-54-279-2150, E-mail: hskim@postech.ac.kr, uptonsong@163.com] Copyright (c) The Korean Institute of Metals and Materials compression, one of the dies is rotated at a set rotational speed and surface friction force, while the high pressure is maintained. A large amount of shear deformation is imposed on a disk-shaped workpiece by multiple rotations of the die during the torsion stage. During the HPT process, the heterogeneity of the deformation is affected by factors such as pressure, the number of revolutions under torsion, the coefficient of friction, and anvil geometry. Previous research has mainly focused on the first two factors [5,10-13].

Recent results indicate that when considering the plastic-deformation and hardness-distribution characteristics of the HPT-processed sample, the compression stage cannot be ignored [14,15]. Heterogeneous plastic deformation was found during the compression stage of the HPT process using the finite element method (FEM) and experimental approaches. The degree of heterogeneous deformation grows gradually with increasing applied pressure: less deformation in the center, and greater deformation at the edge along the radial direction. However, there is an opposite trend, with greater deformation in the center compared to that at the periphery, near the central (i.e., equatorial) plane along the thickness. Furthermore, the strain increases significantly with 

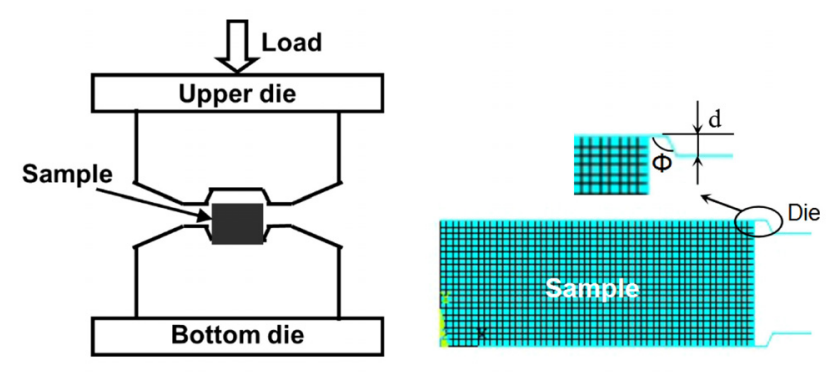

Fig. 1. Anvil structure and the meshed simulation model.

increasing distance from the center, near the surface along the thickness. The friction force affected the effective strain more in the middle and edge regions than in the central region. The experimental and simulation results indicate that the deformation, hardness, and microstructure distributions under different factors were each distinct [10-15]. To the authors' knowledge, the effects of friction and anvil geometry on heterogeneous deformation during the compression stage of the HPT process has not been addressed in the literature.

In this paper, the effects of friction and anvil geometry on the deformation aspects of compressed copper disks during the HPT process were investigated using FEM and experiments.

\section{EXPERIMENT AND SIMULATION PROCEDURE}

Commercial purity copper (99.98 wt \%) was used as raw material for the HPT process. Cylindrical samples $(10.0 \mathrm{~mm}$ diameter, $2.0 \mathrm{~mm}$ thickness) were machined after annealing heat-treatment $\left(600{ }^{\circ} \mathrm{C}\right.$ for $2 \mathrm{~h}$ followed by cooling in a furnace), which resulted in a sample grain size of 20-40 $\mu \mathrm{m}$ and hardness of about $56 \mathrm{HV}$. The upper anvil of the device used for the semi-constrained HPT process was fixed. Surface pressure of $2 \mathrm{GPa}$ was imposed on the disks at room temperature. The time under compression load was set at $10 \mathrm{~s}$.

An FM-700 micro-hardness tester was used to measure the hardness after pressure loading of $100 \mathrm{~g}$ for $10 \mathrm{~s}$. Microstructures of samples from different positions on the disks, taken from the disks after the HPT process, were observed using optical microscopy (Olympus U-TV0.5xc).
Isothermal FEM simulations of the compression stage of the HPT process, were performed using the commercial rigid-plastic FEM code ANSYS. In the FEM simulations, the initial dimensions of the samples were $10.0 \mathrm{~mm}$ diameter and $2.0 \mathrm{~mm}$ thickness. The size of the initial FEM meshes of the samples was $0.1 \mathrm{~mm}$. This size was confirmed to be enough to show local deformation of the sample by calculations which varied the number of elements. In the investigation, three factors: friction coefficient $(\mu)$, depth of the cavity on an anvil (d), and wall angle of cavity $(\Phi)$, were analyzed in the compression stage of the HPT process. The anvil structure is shown in Figure 1.

All the strain data taken from the ANSYS code were processed using the Origin software for linear presentation.

\section{SIMULATION RESULTS AND DISCUSION}

\subsection{Effect of friction on the plastic deformation in the compressive stage}

There have been preliminary investigations on the effect of friction between the anvils and workpiece on plastic deformation during the compression stage of HPT [4,12]. The results indicated that the effective strain remarkably increased with the number of revolutions under torsion, compared to the strain in the compression stage. Although there was little variation in the central region under different friction coefficients, the strain increased significantly with distance from the center, due to frictional shear stress. Furthermore, the friction force influenced the effective strain more remarkably in the central and edge regions of the compressed disks than in the middle region. Systematic investigation of this issue is worthy of deep research because of the important role of friction in the plastic deformation of the disks during the HPT process. Figure 2 shows the cross-sectional planes with effective strain distributions after compression of a copper disk under different conditions: friction coefficients of $0.1,0.5,1.0$, and 3.0; applied pressure of $2 \mathrm{GPa}$; and wall angle of $120^{\circ}$.

The distribution of effective strain in the compressed copper disk is more heterogeneous as the friction coefficient increases. The radial heterogeneity of the effective strain on 

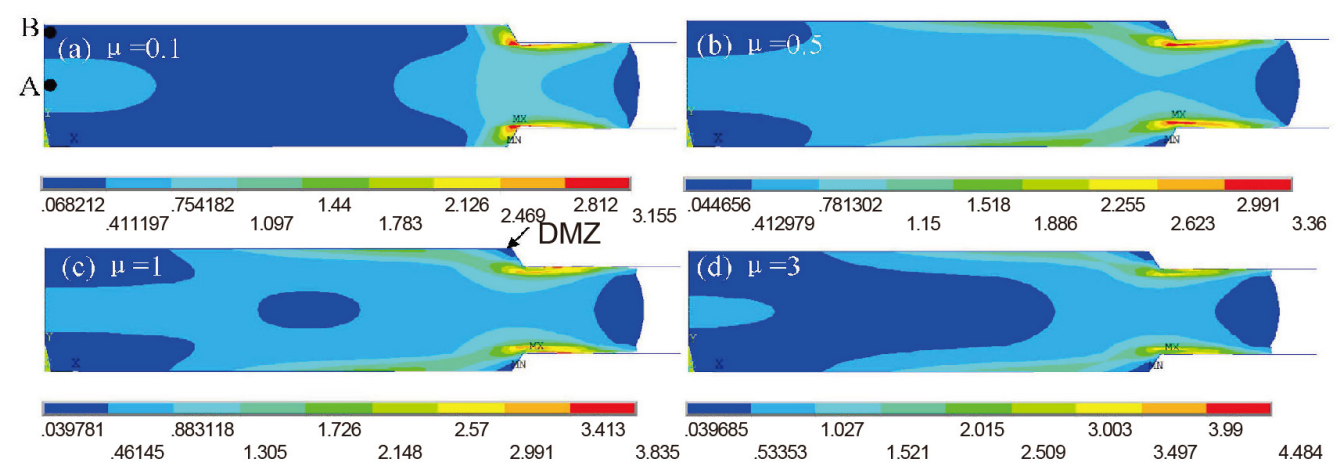

Fig. 2. Effective strain distributions of the half cross-sectional planes at four friction coefficients of (a) 0.1 , (b) 0.5 , (c) 1.0 , and (d) 3.0 under $2 \mathrm{GPa}$ and $120^{\circ}$ wall angle.

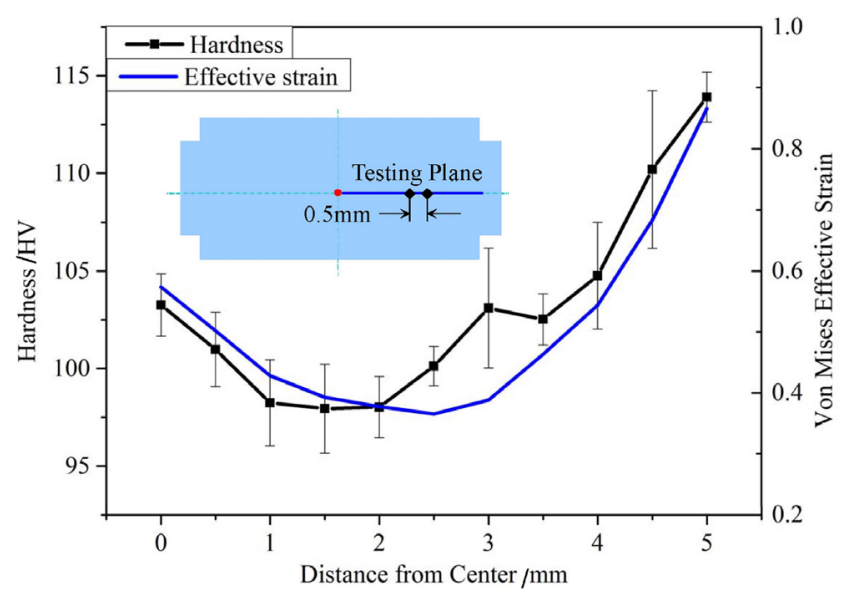

Fig. 3. Path plots of the effective strain and hardness along the radial direction under $2 \mathrm{GPa}, 120^{\circ}$ wall angle and the friction coefficient 0.1 .

the plane is clearly displayed. The effective strain is lower in the center and higher at the edge of the compressed disks, and the effective strain distributions are more and more heterogeneous from center to edge. For example, with the friction coefficient of 3.0, as shown in Figure 2d, the variation of effective strain along the radial direction was from 0.397 in the center to 4.484 at the edge, while with friction coefficient of 0.1 , the effective strain varied from 0.068 to 3.155 , as shown in Figure 2a. This situation of heterogeneous plastic deformation was also reported in the forging process due to friction [16-20]. The results indicate that the fresh area of the contact surface, between the dies and workpiece, increased with increasing friction. Meanwhile, the distribution of the effective strain and hardness became more and more heterogeneous as the friction coefficient increased along the radial and axial directions, which is in good agreement with our results.

More attention should be paid to the strain distribution in the central plane of the thickness direction. The results exhibit a remarkable difference between the top and bottom planes in that the effective strain values at the center are higher than those in other areas (i.e., in the direction indicated by an arrow in Figure 2a). Here, an effective strain of 0.574 occurred in Position A (central plane), while at Position B, near the surface of the upper plane, the effective strain was 0.112. Correspondingly, the hardness values of the two positions were $103.1 \mathrm{HV}$ at $\mathrm{A}$ and $80.5 \mathrm{HV}$ at B. It should be noted that the mechanical properties of the deformed material are attributable to the amount of plastic deformation (i.e., the developments and distributions of strain and stress in the workpiece during the compression process).

The hardness distribution of the experimentally compressed disks was reflected in the strain distribution of the simulations. Figure 3 indicates the trend in variation, along the radial direction. Figure 3 also provides a comparison between experimental hardness based on the average of four groups of experimental data, and simulation results of the effective strain distribution in the compressed copper disk, under the conditions of a friction coefficient of 0.1 , applied pressure of $2 \mathrm{GPa}$, and wall angle of $120^{\circ}$. The same distribution trend was indicated in both the experimental and simulated results. Hence, the reliability of this computer simulation is verified. 

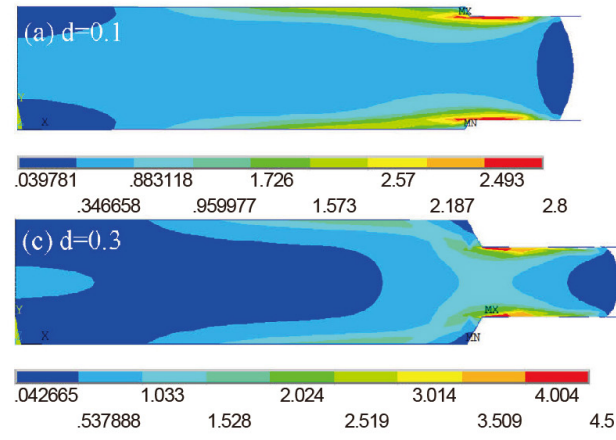
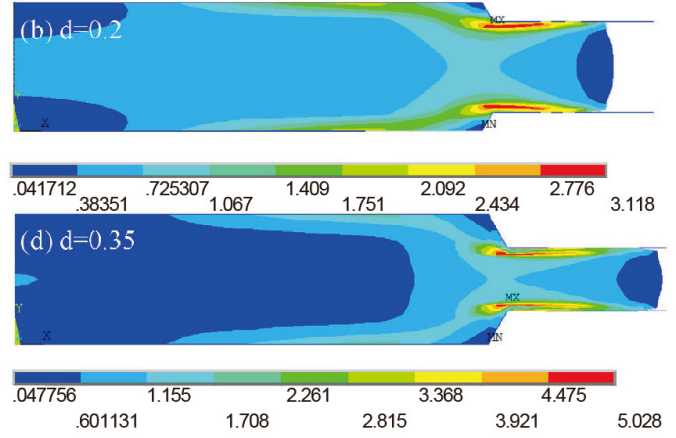

Fig. 4. Effective strain distributions of the half cross-sectional planes at different depths of the cavity: (a) $d=1 \mathrm{~mm}$, (b) $\mathrm{d}=0.2 \mathrm{~mm}$, (c) $\mathrm{d}=0.3 \mathrm{~mm}$, and (d) $\mathrm{d}=0.35 \mathrm{~mm}$.
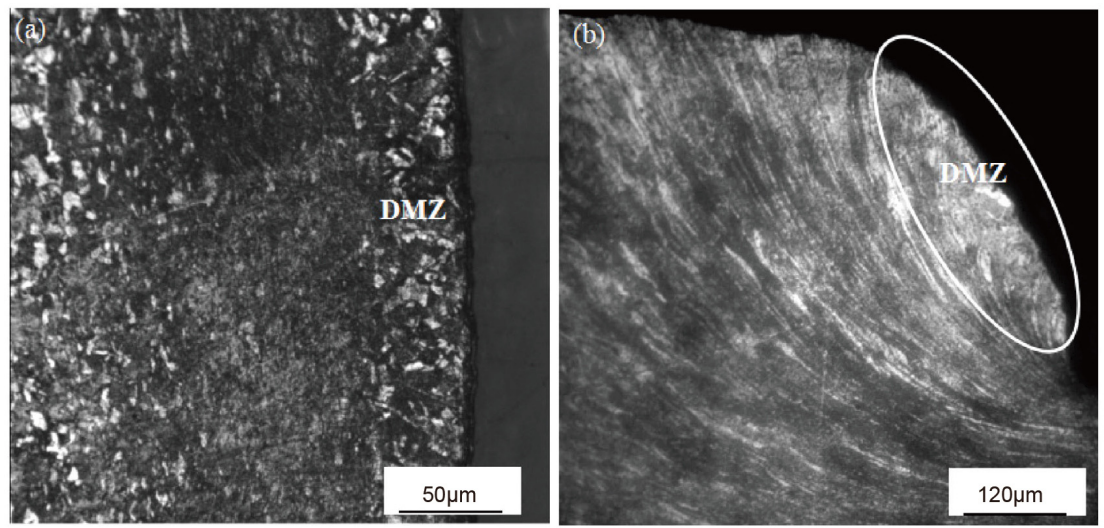

Fig. 5. The microstructure distribution of DMZ on the radial direction (a) and thickness direction (b) of compressed copper disk

\subsection{Effect of friction and anvil cavity structure on the dead metal zone}

The dead metal zone (DMZ) in the HPT process was first reported by Lee et al., and verified after consideration of simulation results and inspections of microstructures from the literature [21]. In the HPT process, plastic deformation increases from the center to the edge in the radial direction of the workpiece. A sticking condition is maintained between the disk and the anvils when the traction is great enough to resist a high friction force. While there is an almost negligible strain rate and strain in the corner region, under high pressure and friction a stagnant zone is generated due to the vertical wall constraint.

In the present work, the simulation results also indicated that an obvious DMZ appeared with an increase in friction coefficient (Figure 2), and the microstructure distribution of $\mathrm{DMZ}$ is shown in Figure 5 with a wall angle of $120^{\circ}$. There is almost no DMZ when the friction coefficient is low $(\mu=0.1$; see Figure 2a). Therefore, it is clear that friction remarkably influences initiation of the DMZ during the plastic deformation process in the compression stage. That is, a DMZ occurs at the corner edge of the disk under a high friction coefficient, not only in the torsion stage, but also in the compression stage of the HPT process.

Another important factor that influences initiation of the $\mathrm{DMZ}$ is the geometry of the anvil cavity, especially the depth of the cavity on the anvil. Figure 4 displays the effective strain distributions on the cross-sectional planes of the compressed disks at different depths of the cavity, under conditions of $2 \mathrm{GPa}$ and a friction coefficient of 0.6 . According to the effective strain distribution of the compressed disk, the degree of heterogeneity and the value of the effective strain increase as the depth of the cavity increases, along the thickness direction. Figure 4 shows lower values of effective strain in the centers and higher values at the edges of the samples. In addition, the length of flash and the area of DMZ increase with the depth of the cavity. 

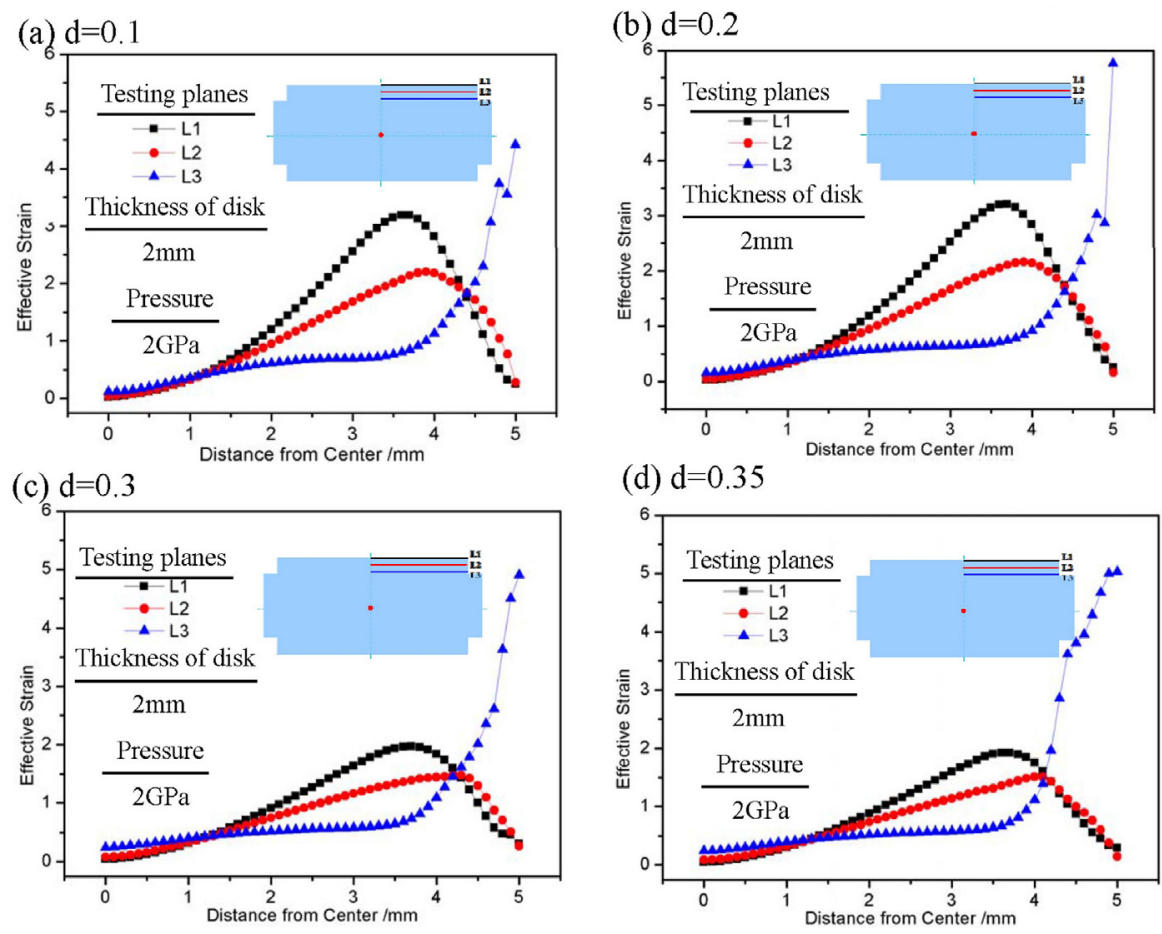

Fig. 6. Path plots of the effective strain on the three lines in the disk at different depths of the cavity: (a) $\mathrm{d}=0.1 \mathrm{~mm},(\mathrm{~b}) \mathrm{d}=0.2 \mathrm{~mm}$, (c) $\mathrm{d}=0.3 \mathrm{~mm}$, and (d) $\mathrm{d}=0.35 \mathrm{~mm}$.
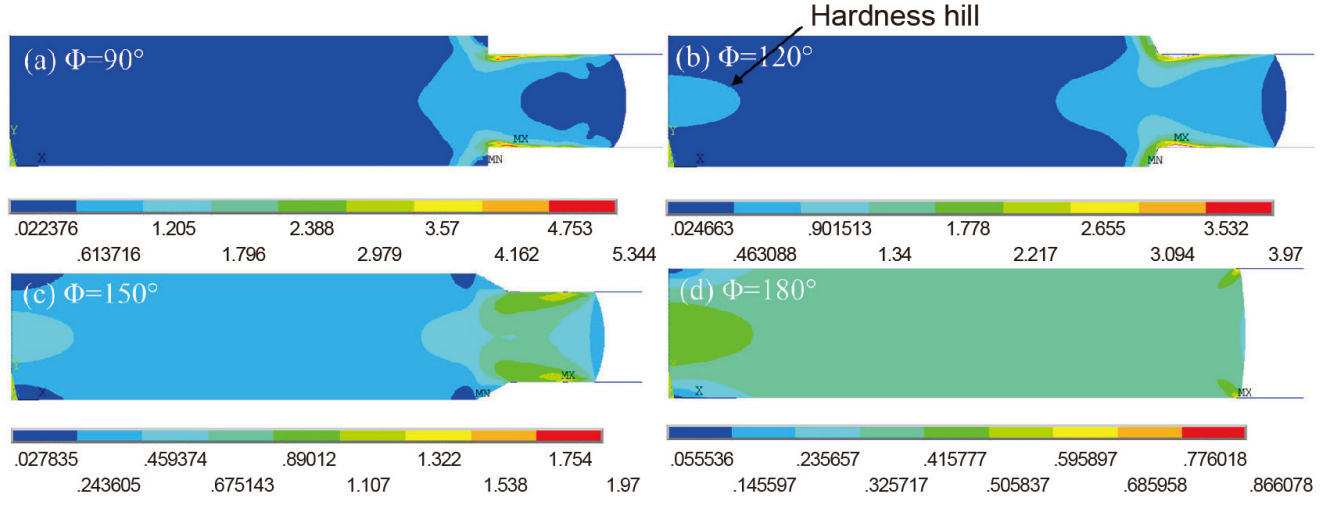

Fig. 7. Effective strain distributions of the half cross-sectional planes at four wall angles of (a) $90^{\circ}$, (b) $120^{\circ}$, (c) $150^{\circ}$, and (d) $180^{\circ}$ under $2 \mathrm{GPa}$ and the friction coefficient 0.1 .

Three lines of the compressed disk (Figure 6) were investigated in relation to DMZ, and the lines L1-L3 were effective-strain path-plot lines equally spaced from each other. The results indicated that the DMZ occurred at the surface corner of the disk and that the compressed disk becomes more heterogeneous from the surface to the central plane. However, the degree of deformation in the surface region of the disk decreased with increasing depth of the cavity.

\subsection{Effect of wall angle of cavity $(\Phi)$}

Figure 7 shows the effective strain distributions of the half cross-sectional planes along the thickness direction of a compressed copper disk, under the conditions of friction coefficient 0.1 , depth $0.2 \mathrm{~mm}$, and applied pressure of $2 \mathrm{GPa}$ without revolution. The results can be described based on two types of HPT, unconstrained $\left(\Phi=180^{\circ}\right)$ and constrained $(\Phi$ $<180^{\circ}$ ) HPT. The plastic deformation in the HPT process increased from the surface plane to the central plane of the 

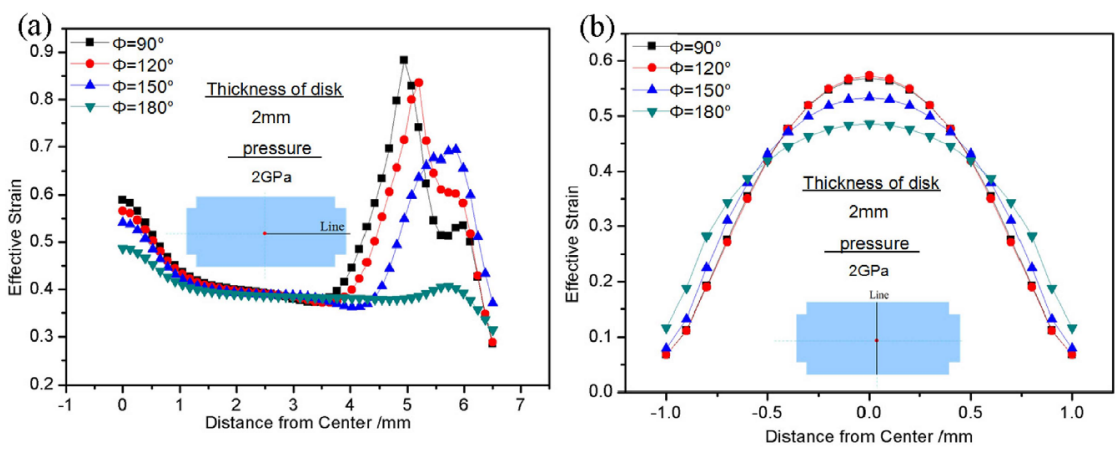

Fig. 8. Path plots of the effective strain on two lines (a central line along the radial direction, b central line along the thickness direction) listed in the disk at four wall angles of the cavity.

disk. The hardness of the central plane was also higher, while the high hardness in the thick central plane as shown in Figure 7, is called a hardness hill in the literature [11,14,22]. The results indicate a variation in the hardness hill as the wall angle of the cavity is increased. However, there was almost no hardness hill at a low angle $\left(\Phi=90^{\circ}\right)$ as shown in Figure 7a. That is, the wall angle of the cavity can remarkably influence the hardness hill during the plastic deformation process of the compression stage.

In addition, the area of the DMZ decreased with increasing wall angle due to the vertical wall constraint under high pressure, as clearly seen in Figure 3, and it dropped to zero when the value of the wall angle increased to $180^{\circ}$, which means there is no DMZ on the disk in the unconstrained HPT processing. There was only a small variation in effective strain, and the hardness was generally homogeneous in the radial middle zones of the disks; however, a large variation occurred at the edge of the disk. Two lines were compared to determine the distribution of effective strain, and are presented in Figure 8.

Figure 8 indicates that the plastic deformation was inhomogeneous along the thickness direction of the disk. Although the area of the hardness hill increased as the wall angle increased, the maximum effective strain in the area of the hardness hill decreased as the angle increased, as shown in Figure 8b. There was significant plastic deformation when the wall angle was less than $180^{\circ}$ (indicated in Figure 8a), while the plastic deformation was relatively uniform along the radial direction under the wall angle condition of $180^{\circ}$. In addition, the anvil geometry also affected the flash of the disk. Interestingly, there was little variation in the flash length of the compressed disk at different wall angles, although a large distinction of strain in the flash regions happened. In any case, the wall angle of the cavity plays an important role in the heterogeneous deformation of the main body of the workpiece.

\section{CONCLUSIONS}

In this study, the plastic deformation behavior of copper disks at the early stage of the HPT process was investigated. Heterogeneous strain and variation in hardness (lower hardness in the center and higher hardness at the edge), exist on the thickness-direction planes of disks, in relation to details of the die-workpiece friction and anvil gap geometry. There is large variation in the flash of the compressed disks under the influence of a series of parameters. According to the results and analysis, there are two effects on the plastic deformation characteristics of the compressed disks resulting from the friction and anvil geometry: the first has two aspects, the heterogeneous level of strain distribution along the thickness and along the radial directions; the other is flash.

The results indicate that the degree of heterogeneous deformation increases as the depth of the cavity and friction coefficient increase. There is less effective strain in the center and higher strain at the edge of the compressed disks, while heterogeneity decreases along the thickness direction with increase in the wall angle. This situation is more extreme in 
the central region than in the middle radius region. Two special regions: the hardness hill and dead metal zones, were investigated. The area of the hardness hill increases with increasing wall angle and cavity depth, while the maximum effective strain in the area of the hardness hill decreases as the angle increases (within an appropriate range). The area of the dead-metal zone decreases as the wall angle increases, and increases with cavity depth. In addition, the length of the flash increases with cavity depth, but shortens most at the wall angle of $180^{\circ}$. Thus, the friction coefficient and anvil geometry play important roles in heterogeneous deformation during the initial stage of the HPT process.

\section{ACKNOWLEDGMENTS}

Y. P. Song acknowledges the postdoctoral fellowship supported by the National Research Foundation through the Korea-China Young Scientists Program, Korea. Furthermore, this work was also supported by Chinese National Fusion Project for ITER (NO. 2013GB110005), Innovation team fund for fruit industry of modern agricultural technology system in Shandong Province (SDAIT-06-12), Research project-2015 on intelligent agricultural mechanization equipment of Shandong Agricultural University, Special project for independent innovation of Shandong province (2013CXC90201). This work was supported by the National Research Foundation of Korea (NRF) grant funded by the Korea government (MSIP) (No. 2014R1A2A1A10051322).

\section{REFERENCES}

1. R. Z. Valiev, R. K. Islamgaliev, and I. V. Alexandrov, Progr. Mater. Sci. 45, 103 (2000).

2. S. J. Yoo and W. J. Kim, Korean J. Met. Mater. 52, 561
(2014)

3. R. Z. Valiev and T. G. Langdon, Prog. Mater. Sci. 51, 881 (2006).

4. A. P. Zhilyaev and T. G. Langdon, Prog. Mater. Sci. 53, 893 (2008).

5. R. B. Figueiredo, M. T. Aguilar, P. R. Cetlin, and T. G. Langdon, Metall. Mater. Trans. A 42, 3013 (2011).

6. A. Hohenwarter, A. Bachmaier, B. Gludovatz, S. Scheriau, and R. Pippan, Int. J. Mater. Res. 100, 1653 (2009).

7. A. P. Zhilyaev, K. Oh-ishi, T. G. Langdon, and T. R. McNelley, Mater. Sci. Eng. A 410-411, 277 (2005).

8. H. S. Kim, S. H. Joo, and H. J. Jeong, Korean J. Met. Mater. 52, 87 (2014).

9. H. S. Kim, W. S. Ryu, M. Janecek, S. C. Baik, and Y. Estrin, Adv. Eng. Mater. 7, 43 (2005).

10. Y. P. Song, W. K. Wang, D. S. Gao, H. S. Kim, E. Y. Yoon, D. J. Lee, C. S. Lee, and J. Guo, Materials Sciences and Applications 3, 234 (2012).

11. Y. P. Song, W. K. Wang, D. J. Lee, H. J. Jeong, S. Lee, and H. S. Kim, Met. Mater. Int. 21, 7 (2015).

12. Y. P. Song, W. K. Wang, D. S. Gao, E. Y. Yoon, D. J. Lee, and H. S. Kim, Met. Mater. Int. 20, 445 (2014).

13. W. K. Wang, Y. P. Song, E. Y. Yoon, D. J. Lee, J. H. Lee, and H. S. Kim, Met. Mater. Int. 19, 1021 (2013).

14. Y. P. Song, W. K. Wang, D. S. Gao, E. Y. Yoon, D. J. Lee, C. S. Lee, and H. S. Kim, J. Mater. Sci. 48, 4698 (2013).

15. Y. P. Song, E. Y. Yoon, D. J. Lee, J. H. Lee, and H. S. Kim, Mater. Sci. Eng. A. 528, 4840 (2011).

16. Z. Horita, and T. G. Langdon, Scripta Mater. 58, 1029 (2008).

17. T. D. Kil, S. W. Han, and Y. H. Moon, Korean J. Met. Mater. 52, 731 (2014).

18. R. G. Narayanan, M. Gopal, and A. Rajadurai, J. Test. Eval. 36, 1 (2008).

19. S. Q. Xu, Z. Y. Chen, and S. Y. Zhang. Forging and Stamping Technology (in Chinese) 29, 46 (2004).

20. P. Lu, G. Q. Zhao, Y. J. Guan, and J. L. Cheng. China Metal Forging Equipment and Manufacturing Technology (in Chinese). 43, 75 (2008).

21. D. J. Lee, E. Y. Yoon, L. J. Park, and H. S. Kim, Scripta Mater. 67, 384 (2012).

22. Y. P. Song, W. K. Wang, D. S. Gao, J. H. Lee, E. Y. Yoon, D. J. Lee, C. S. Lee, and H. S. Kim, Rare Metal Mat. Eng. 42(S2), 301 (2013). 